\title{
Identification of minor inner-membrane components of the Shigella type III secretion system 'needle complex'
}

\author{
Correspondence \\ Ariel J. Blocker \\ ariel.blocker@path.ox.ac.uk
}

Received 6 March 2007

Revised 16 April 2007

Accepted 24 April 2007

\author{
Sebastian F. Zenk, ${ }^{1} \dagger$ David Stabat ${ }^{1} \ddagger$ Julie L. Hodgkinson, ${ }^{1}$ \\ Andreas K. J. Veenendaal, ${ }^{1}$ Steven Johnson ${ }^{1,2} \S$ and Ariel J. Blocker ${ }^{1}$
${ }^{1}$ Sir William Dunn School of Pathology, University of Oxford, South Parks Road, Oxford OX1 3RE, UK
${ }^{2}$ Laboratory of Molecular Biophysics, Department of Biochemistry, University of Oxford, South Parks Road, Oxford OX1 30U, UK

\begin{abstract}
Type III secretion systems (T3SSs or secretons) are central virulence factors of many Gram-negative bacteria, used to inject protein effectors of virulence into eukaryotic host cells. Their overall morphology, consisting of a cytoplasmic region, an inner- and outer-membrane section and an extracellular needle, is conserved in various species. A portion of the secreton, containing the transmembrane regions and needle, has been isolated biochemically and termed the 'needle complex' (NC). However, there are still unsolved questions concerning the nature and relative arrangement of the proteins assembling the NC. Until these are resolved, the mode of function of the NC cannot be clarified. This paper describes an affinity purification method that enables highly efficient purification of Shigella NCs under near-physiological conditions. Using this method, three new minor components of the NC were identified by mass spectrometry: IpaD, a known component of the needle tip complex, and two predicted components of its central inner-membrane export apparatus, Spa40 and Spa24. A further minor component of the NC, MxiM, is only detected by immunoblotting. MxiM is a 'pilotin'-type protein for the outer-membrane 'secretin' ring formed of MxiD. As expected, it localized to the outer rim of the upper ring of NCs, validating the other findings.
\end{abstract}

\section{INTRODUCTION}

Shigella flexneri is the causative agent of bacillary dysentery in humans (Hale, 1998). Shigella uses a type III secretion system (T3SS) as its major virulence device for invasion of, and dissemination within, the gut epithelial lining (Cossart \& Sansonetti, 2004). T3SSs, which are found in many other Gram-negative bacterial pathogens, serve as molecular injection devices to deliver bacterial virulence effector

†Present address: Institut für Klinische Mikrobiologie, Immunologie und Hygiene, FAU Erlangen-Nürnberg, Wasserturmstraße 3-5, D-91054 Erlangen, Germany.

$\ddagger$ Present address: Université Pierre et Marie Curie, Paris VI, 4 place Jussieu, 75252, Paris cedex 03, France.

§Present address: Sir William Dunn School of Pathology, University of Oxford, South Parks Road, Oxford OX1 3RE, UK.

Abbreviations: CR, Congo red; DDAO, n-dodecyl- $N, N$-dimethylamine- $N$ oxide; EM, electron microscopy; i ${ }^{6} A 37$, tRNA $N^{6}$-isopentenyladenosine synthetase; LAPAO, 3-lauroamido- $N, N^{\prime}$-dimethyl-3-n-propylamine oxide; $\mathrm{NC}$, needle complex; OG, n-octyl $\beta$-D-glucopyranoside; $\mathrm{Pl}$, protease inhibitor; PTA, phosphotungstic acid; T3SS, type III secretion system; TX100, Triton X-100.

Supplementary material is available with the online version of this paper. proteins into eukaryotic host target cells. They are genetically, morphologically and structurally related to the basal bodies of bacterial flagella (Blocker et al., 2003).

The Shigella type III secreton is encoded on a $31 \mathrm{~kb}$ fragment of a large virulence plasmid. About 25 genes within the mxilspa operons encode proteins needed for the assembly of the secreton (Parsot, 1994). This apparatus is made of three major parts (Blocker et al., 1999): a cytoplasmic region known as the 'bulb', of which Spa33 is a component (Morita-Ishihara et al., 2006), a region spanning both bacterial membranes and an extracellular 'needle'. A part of the machinery, containing the hollow needle $(\sim 10 \times 60 \mathrm{~nm})$ and some of the transmembrane assembly (Blocker et al., 1999; Kubori et al., 1998), has been isolated and termed the 'needle complex' (NC). Five main components of the Shigella NC have been identified. MxiG and MxiJ are located in the inner membrane (Allaoui et al., 1992, 1995) and form a double ring structure. The largely periplasmic MxiJ protein, carrying a single Cterminal transmembrane helix and lipidated at its $\mathrm{N}$ terminus, is located at the centre of the inner-membrane ring and surrounded by MxiG (Allaoui et al., 1992; Yip et al., 2005). MxiD is inserted in the outer membrane, 
forming a 'secretin' ring (Allaoui et al., 1993; Burghout et al., 2004). MxiD's 'pilotin', MxiM, an outer-membrane lipoprotein required for efficient secreton assembly (Schuch \& Maurelli, 1999, 2001), was also recently identified in NC preparations, but its final localization within the structure is unclear (Sani et al., 2007a). MxiH is the protein that forms the extracellular part of the needle (Blocker et al., 2001) and its periplasmic continuation is likely built up by the sequence-related MxiI (Marlovits et al., 2004, 2006).

The stoichiometry of protein components of the NC has been approximated to 120 subunits of $\mathrm{MxiH}, 5-10$ subunits of MxiI and 19-24 copies each of MxiG and MxiJ and possibly MxiD (Marlovits et al., 2004; Yip et al., 2005). However, there are a number of proteins, encoded by the ipa, $m x i$ and spa operons, that are predicted to form inner-membrane as well as external parts of the secreton, but have never been identified in NC preparations. We wanted to investigate the presence of these likely minor components. For the future, we also need to improve the resolution of NC image reconstructions beyond $15 \AA$ (1.5 nm) (Blocker et al., 2001; Marlovits et al., 2004) for integration with our analyses of needle structure and function (Cordes et al., 2003, 2005; Kenjale et al., 2005; Deane et al., 2006; Veenendaal et al., 2007). To these aims, we designed a novel, nickel-affinity based purification strategy for the NC. An N-terminal $6 \times$ His-tag was introduced into MxiG expressed from an inducible promoter in $m x i G$-deficient Shigella. This led to synthesis of functional secretons carrying multiple $6 \times$ His-tags. The new purification method generates acceptable yields of NCs, which display very high purity. This degree of enrichment is now sufficient to allow detection of several of the expected minor components.

\section{METHODS}

Bacterial strains and growth conditions. S. flexneri strains used in this work are listed in Supplementary Table S1 (available with the online version of this paper). Bacteria were grown as previously described (Blocker et al., 1999).

Plasmids. $m x i G$ was cloned into the pBAD/MycHisA expression vector system via the intermediate PCRII TOPO vector (Invitrogen). The design of the primers and the nomenclature for constructs generated are detailed in Supplementary Tables S3 and S2, respectively. The $\mathrm{N}$-terminal $6 \times$ His-tagged MxiG construct encoded the sequence MGHHHHHHGSR followed by the full-length mxiG sequence, giving rise to pSZ1. The $3^{\prime} \mathrm{KpnI}$ site used was located immediately downstream of the stop codon. pSZ2, encoding the Cterminal $6 \times$ His-tagged MxiG, was produced with the $5^{\prime} \mathrm{NcoI}$ site and a $3^{\prime} B s t$ BI site followed by a downstream $m y c^{-}$and $6 \times$ His-tags. pSZ3 (pBAD/MycHisA : $m x i G$ ) is the $B g l \mathrm{II} / K p n \mathrm{I}$ precursor of pSZ1 lacking the $6 \times$ His-tag and hence referred to as the untagged version of MxiG. This construct carries the full-length mxiG sequence, lacking only its native start codon, but in-frame with the start codon at the beginning of the multiple cloning site of the $\mathrm{pBAD} / \mathrm{M} y \mathrm{cHisA}$ vector. The recombinant $\mathrm{MxiG}$ expressed from $\mathrm{pSZ3}$ therefore contains five additional amino acids at its $\mathrm{N}$ terminus: aspartic acid, proline, two serines and an arginine.
Overnight leakage and Congo red induction. These assays were carried out according to established protocols (Kenjale et al., 2005).

Generation of monoclonal antibodies against native NCs. These antibodies were generated according to the method of Niebuhr \& Ebel (2003). Briefly, female Balb/C mice were immunized with NCs, purified as previously described (Blocker et al., 2001) and mixed with complete Freund's adjuvant, six times at 3-day intervals subcutaneously in one of their hind legs, prior to isolation of the lymphocytes from their popliteal lymph nodes. These were then used for PEGinduced fusion with myeloma cells (P3-X63-Ag8; ATCC CRL 1580). After isolation by limiting dilution and selection, culture supernatant from single clones was screened, in 96-well plates by ELISA, on wildtype and NC component mutant total Shigella extracts. Promising clones were further subcloned to ensure homogeneity and rescreened by Western blotting on total extracts of Escherichia coli strains expressing single known NC component genes. Only anti-MxiG and anti-MxiJ antibodies have been isolated in this fashion so far, but most initial clones remain available for further testing.

NC affinity purification. This was performed largely as in Blocker et al. (2001), with the following modifications. Bacteria (200 ml) were grown from overnight subcultures to $\mathrm{OD}_{600} 1$ in presence of the necessary antibiotics and $0.02 \%(\mathrm{w} / \mathrm{v})$ arabinose, shaking at $37^{\circ} \mathrm{C}$. They were collected by centrifugation at $2200 \mathrm{~g}$ for $7 \mathrm{~min}$ at $4{ }^{\circ} \mathrm{C}$, washed once in cold PBS and resuspended in $5 \mathrm{ml} 0.5 \mathrm{M}$ sucrose, $100 \mathrm{mM}$ Tris, $\mathrm{pH}$ 8. Cells were microscopically checked for complete spheroplasting, after addition of $1 \mathrm{mM}$ EDTA and $1 \mathrm{mg}$ lysozyme $\mathrm{ml}^{-1}$ (in a $500 \mu \mathrm{l}$ stock solution), and incubation in a $37^{\circ} \mathrm{C}$ waterbath for up to $1 \mathrm{~h}$. Prior to lysis, an EDTA-free protease inhibitor (PI) cocktail (Mini-Complete; Roche) was added. For lysis, various detergents (always from freshly made stocks) were alternatively added, as $1 \mathrm{ml} 10 \%$ or $20 \%$ Triton X-100 (TX-100; Sigma-Aldrich) or $10 \%$ n-octyl $\beta$-D-glucopyranoside, 3-lauroamido- $N, N^{\prime}$-dimethyl3-n-propylamine oxide or $\mathrm{n}$-dodecyl- $\mathrm{N}, \mathrm{N}$-dimethylamine- $\mathrm{N}$-oxide (OG, LAPAO and DDAO, respectively; Anatrace). Samples were left to solubilize for $30 \mathrm{~min}$ at $4{ }^{\circ} \mathrm{C}$. Released DNA was digested with DNase I in the presence of $10 \mathrm{mM} \mathrm{MgSO}_{4}$ (added as a $1 \mathrm{ml}$ stock solution, fraction 1). The solution was then cleared by centrifugation $(20 \mathrm{~min}, 20500 \mathrm{~g}$ ). The pellet (fraction 3) was adjusted to the same $7.5 \mathrm{ml}$ volume as the supernatant (fraction 2) to facilitate later analysis. The supernatant was subjected to further centrifugation at $94000 \mathrm{~g}$ for $2 \mathrm{~h}$ (supernatant is fraction 4). A thorough resuspension of pelleted NCs (fraction 5) was carried out in $7.5 \mathrm{ml}$ of $5 \mathrm{mM}$ imidazole, $150 \mathrm{mM} \mathrm{NaCl}, 10 \mathrm{mM}$ Tris, $\mathrm{pH} \mathrm{8,0.5 \%} \mathrm{detergent,}$ EDTA-free PI using a small volume and a plastic Pasteur pipette initially and then 10 passes through a $5 \mathrm{ml}$ syringe with a 26 gauge needle. The solution was then added to $160 \mu \mathrm{l}$ of $50 \%$ Ni-NTA agarose beads slurry (Qiagen), previously washed in resuspension buffer, and left rotating overnight in a $15 \mathrm{ml}$ tube at $4{ }^{\circ} \mathrm{C}$. According to the manufacturer, Ni-NTA beads are able to bind $50-100 \mu \mathrm{g}$ of His-tagged protein per $20 \mu$ l. Up to an eightfold excess of beads was added relative to the total amount of $\mathrm{His}_{6}-\mathrm{MxiG}$ present in the preparation (estimated at $\sim 20$ copies per NC and $\sim 100$ NCs per bacterial cell). However, we were never able to obtain more than $50 \%$ depletion of the total $\mathrm{His}_{6}$-MxiG during the overnight binding step (compare the lower panels on the left and right side of Fig. 2, especially fractions 5 and 6, which are the resuspended NC pellet, before and after incubation with the beads). Adding more beads resulted in higher unspecific protein background in the elution. We compromised in not repeating the binding step with fresh beads because we found that the entire protocol had to be performed as quickly as possible to limit structural damage to NCs. Afterwards, the beads were pelleted via centrifugation at $1000 \mathrm{~g}, 10 \mathrm{~min}$ (supernatant is fraction 6), washed briefly with $7.5 \mathrm{ml}$ washing buffer $1(50 \mathrm{mM}$ imidazole, $150 \mathrm{mM} \mathrm{NaCl}, 10 \mathrm{mM}$ Tris, $\mathrm{pH} \mathrm{8,} \mathrm{0.1 \%} \mathrm{N-lauroylsarco-}$ sine and EDTA-free PI) and washing buffer 2 (containing no 
$N$-lauroylsarcosine, but instead $0.1 \% \mathrm{TX}-100$ ), forming fractions 7 and 8 , respectively. Finally, NCs were eluted using two aliquots of $100 \mu$ l elution buffer ( $500 \mathrm{mM}$ imidazole, $0.1 \%$ TX-100, $10 \mathrm{mM}$ Tris, pH 8, PIs), shaking on ice for $30 \mathrm{~min}$ and collected using a pulled glass Pasteur pipette with a diameter small enough to exclude the beads (NCs). EDTA was added to a final concentration of $1 \mathrm{mM}$. The beads (B) were resuspended in $200 \mu \mathrm{l}$ SDS-PAGE sample buffer. It was found that the protocol could only be satisfactorily scaled up by keeping all proportions and incubation conditions identical and pooling samples at the stage of incubation with the beads.

NC size-exclusion chromatography and storage. For sizeexclusion chromatography, large-scale isolations were performed (1-2 1 of bacterial cultures). Affinity-purified NCs were concentrated to $100 \mu \mathrm{l}$ (Ultrafree-0.5 Centrifugal Filter Device, $5 \mathrm{kDa}$ cut-off; Millipore) and applied to a Superdex S200 HR10/30 gel filtration column (Amersham-Pharmacia) equilibrated in $25 \mathrm{mM}$ Tris $\mathrm{pH} 8$, $2 \mathrm{mM}$ EDTA and $0.1 \%$ detergent. Although we tested filtration matrices optimized for separation of large protein complexes and viruses, such as Sephacryl 1000 (Amersham-Pharmacia), the poorer resolution of these columns meant that the NCs could not be easily or efficiently recovered (not shown). The NCs eluted in the $1 \mathrm{ml}$ fraction corresponding to the void volume of the column and were again concentrated to $<100 \mu \mathrm{l}$. Samples retained their morphology best if the entire purification procedure was performed within 1.5 to 2 days. Finally, it was found that in such purified preparations, NCs preserved their morphology for maximally a couple of days on ice at $4{ }^{\circ} \mathrm{C}$. However, after addition of $10 \% \mathrm{w} / \mathrm{v} \mathrm{D}-(+)$-trehalose (Sigma-Aldrich) as a cryo-preservative (Hirai et al., 1999), they could be flash frozen in tiny aliquots in liquid nitrogen and then stored for months, either at $-80{ }^{\circ} \mathrm{C}$ or in liquid nitrogen.

Electron microscopy (EM). Formvar (300 mesh; Agar Scientific) and carbon-coated glow-discharged copper grids were coated with $4 \mu$ isolated NC solution (appropriately diluted in the size-exclusion chromatography buffer, with or without detergent, mentioned above), washed twice in distilled water, and subsequently stained for 1 min with $2 \%$ phosphotungstic acid (PTA), pH 7.0. EM images were taken on a Philips Tecnai T12 transmission electron microscope at $80 \mathrm{kV}$. Micrographs were recorded at $20000 \times$ magnification on a $2 \mathrm{~K}$ ProScan CCD camera using the SIS software.

Anti-MxiM antibody affinity purification and NC immunogold labelling. Recombinant MxiM was purified as described by Schuch \& Maurelli (1999). A gel filtration step on a Superdex 200 10/30 HR column (Amersham-Pharmacia), equilibrated in $0.1 \mathrm{M} \mathrm{NaHCO}_{3}$ $\mathrm{pH} 8.3$ with $0.5 \mathrm{M} \mathrm{NaCl}$, was used to remove higher and lower molecular mass contaminants. The anti-MxiM rabbit polyclonal antiserum, which we affinity-purified, was initially generated by Schuch \& Maurelli (1999). It was processed as described by Veenendaal et al. (2007) for the anti-IpaD antiserum and its MxiM mono-specificity was then verified by immunoblotting (Supplementary Fig. S1, available with the online version of this article). For immunogold labelling, NCs (derived from the final purification step described above) were diluted $1: 3$ in $\mathrm{NC}$ gel filtration buffer containing $0.1 \%$ Triton X-100 (GF buffer). Copper EM grids (Athene, 400 mesh; Agar Scientific), coated with thin carbon and glow-discharged in argon, were placed for 1 min onto $10 \mu$ of diluted NCs. Grids were then incubated on $10 \mu$ l of primary $\mathrm{Ab}$ mixture (at $0.2 \mathrm{mg} \mathrm{ml}^{-1}$ ), diluted 12-fold with GF buffer. Finally, grids were incubated on $10 \mu \mathrm{l}$ of $5 \mathrm{~nm}$ gold-conjugated goat antirabbit IgG (BBInternational) diluted 1:40 from a $30 \mu \mathrm{g} \mathrm{ml}^{-1}$ stock. As our aim was to precisely localize MxiM, non-saturating concentrations of both primary and gold-coupled secondary Abs were used to allow optimal spatial resolution. All incubations were carried out on ice for 20 min. Between each incubation step excess mixture was drawn off using filter paper and the grids were rinsed in three changes of GF buffer. Finally, grids were stained for $30 \mathrm{~s}$ with $2 \%$ PTA at pH 7.0. Micrographs were taken at 30000 or $49000 \times$ magnification on Kodak 4489 film.

\section{RESULTS}

\section{His $_{6}-$ MxiG functionally complements mxiG $^{-}$ Shigella}

Of all the known NC components, MxiG was selected for His-tagging because it was known to lie within the inner membrane ring of the NC and to be present in 19-24 copies (Blocker et al., 2001; Marlovits et al., 2004; Yip et al., 2005). The atomic structure of the enteropathogenic E. coli homologue of MxiJ, EscJ, the size of the ring it formed, and biotinylation accessibility studies of the Salmonella SPI1 NC (Yip et al., 2005), suggested that MxiG would lie on the outside of the MxiJ ring, with its $\mathrm{N}$ terminus pointing away from the NC core. However, as the relative accessibility of a His-tag at either terminus of MxiG for nickel affinity purification could not be predicted, both $\mathrm{N}$ - and Cterminally His-tagged MxiG, encoded by pSZ1 and pSZ2, respectively, as well as the untagged MxiG, encoded by pSZ3, were made (see Methods and Supplementary material). These plasmids were introduced into an $m x i G^{-}$strain. The resulting strains are hereafter referred to as $m x i G^{-} / \mathrm{N}-\mathrm{His}_{6}-m x i G, \quad m x i G^{-} / \mathrm{C}-\mathrm{His}_{6}-m x i G$ and $m x i G^{-} / m x i G$, respectively.

The expression level of tagged MxiG in $m x i G^{-} / \mathrm{N}-\mathrm{His}_{6^{-}}$ $m x i G$ was compared to the level of the protein in wild-type Shigella by Western blotting. In $m x i G^{-} / \mathrm{N}-\mathrm{His}_{6}-m x i G$, a concentration of $0.02 \%$ arabinose resulted in a $\mathrm{His}_{6}$-MxiG level comparable to that found in wild-type bacteria (Fig. 1a). $\mathrm{His}_{6}$-MxiG displayed the higher molecular mass expected to result from the additional amino acids of the tag. In addition, some $\mathrm{His}_{6}-\mathrm{MxiG}$ was proteolytically cleaved (to a shorter than wild-type MxiG form) or the product of an unidentified internal initiation site upon overexpression (with 0.2 and $2 \%$ arabinose) was observed, as indicated by comparison of blots obtained with an antiMxiG antibody and nickel-labelled detection reagent.

To assess assembly and basal functionality of the T3SS of the complemented $m x i G^{-}$strains, these were tested for overnight 'leakage'. Overnight leakage is defined as a slow and uninduced release of Ipa proteins (the early effectors of Shigella virulence), which occurs during the assembly of the secreton (Magdalena et al., 2002). In the presence of $0.02 \%$ arabinose, both $m x i G^{-} / m x i G$ and $m x i G^{-} / \mathrm{N}-\mathrm{His}_{6}-m x i G$ showed leakage of Ipa proteins. Their Ipa secretion pattern corresponded approximately to that seen in the wild-type strain, but was substantially enhanced in $m x i G^{-} / m x i G$ (Fig. 1b). Strongly altered or absent T3S was detectable in strains expressing either untagged $\mathrm{MxiG}$ or $\mathrm{His}_{6}-\mathrm{MxiG}$ with 0.2 or $2 \%$ arabinose (not shown). These conditions also inhibited cell growth (data not shown) and/or secretion of SepA and/or IcsA, two proteins exported by systems other than T3SS (Benjelloun-Touimi et al., 1995; 
(a)
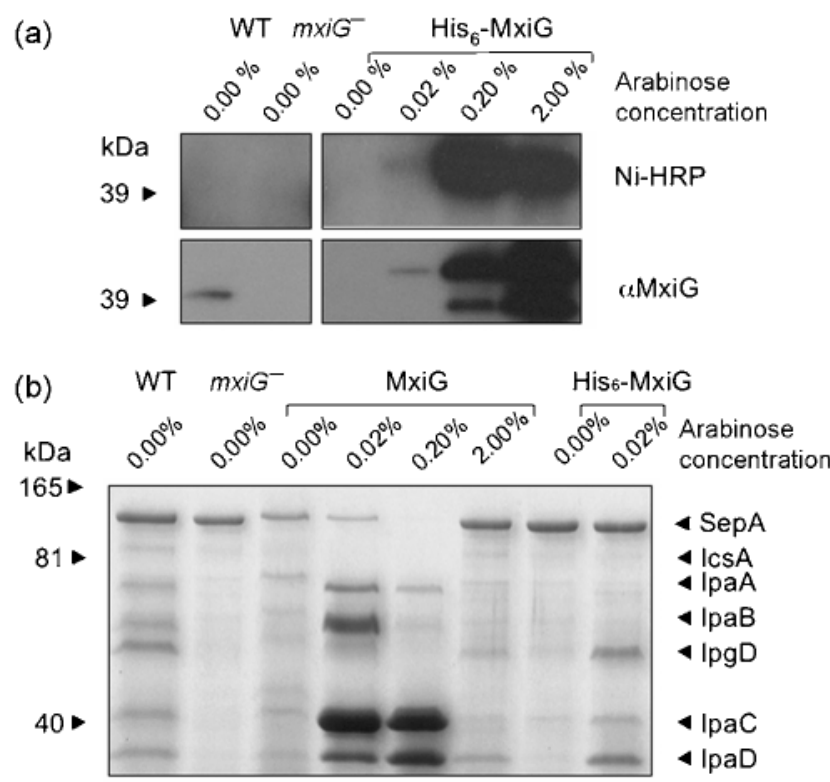

(c)

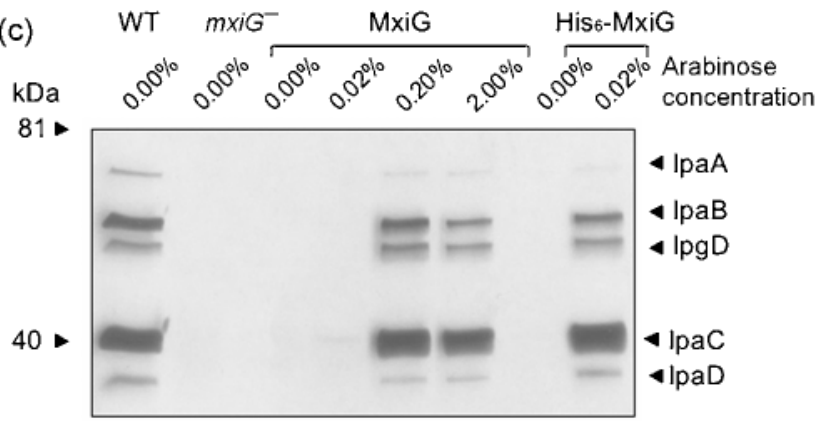

Fig. 1. Levels of MxiG expression, overnight leakage and $C R$ induction of Ipa proteins in the complemented mxiG ${ }^{-}$strains. (a) Detection of $\mathrm{N}$-terminally $\mathrm{His}_{6}$-tagged MxiG and wild-type MxiG in whole-cell lysates of wild-type (WT), $m x i G^{-}$and $m x i G^{-} / \mathrm{N}-\mathrm{His}_{6}{ }^{-}$ $m x i G$ ( $\left.\mathrm{His}_{6}-\mathrm{MxiG}\right)$ Shigella 5 a strains by Western blotting with a nickel-labelled horseradish peroxidase conjugate (Ni-HRP; Pierce) and a self-generated mouse monoclonal anti-MxiG antibody $(\alpha \mathrm{MxiG})$ after induction with different concentrations of arabinose. (b) Overnight leakage of wild-type, $m x i G^{-}, m x i G^{-} / m x i G$ expressing wild-type $m x i G$ (MxiG) as well as $m x i G^{-} / \mathrm{N}-\mathrm{His}_{6}-m x i G$. Bacteria were grown overnight in different concentrations of arabinose and without the inducer as indicated. Culture supernatants were collected, separated by SDS-PAGE and silver stained. (c) The same strains as in (b) were subcultured into exponential phase, washed in PBS and induced with $\mathrm{CR}$ at $37^{\circ} \mathrm{C}$. Supernatants were collected, separated by SDS-PAGE and silver stained. Bacterial pellets or supernatants were adjusted to equivalent cell numbers so each experimental point is directly comparable within each panel. All samples were separated by electrophoresis on 10 or $12 \%$ SDS-polyacrylamide gels.

Suzuki et al., 1995), and hence were assumed to be toxic to cells.

The ability of exponentially growing bacteria to be induced to secrete Ipa proteins by the small amphipathic dye Congo red (CR) verifies the presence of a functional T3SS (Bahrani et al., 1997), in that it is assumed to mimic activation of secretion by host-cell contact. $m x i G^{-} / \mathrm{N}-\mathrm{His}_{6}{ }^{-}$ $m x i G$, when grown to mid-exponential phase in $0.02 \%$ arabinose, was able to release Ipa proteins upon induction, whereas $m x i G^{-} / m x i G$ required at least $0.2 \%$ arabinose to do so (Fig. 1a). Once again, growth of $m x i G^{-} / \mathrm{N}-\mathrm{His}_{6}{ }^{-}$ $m x i G$ in $2 \%$ arabinose was severally inhibited, as was, but to a lesser extent, that of $m x i G^{-} / m x i G$ (not shown). The growth properties and secretion profiles of $m x i G^{-} / \mathrm{C}-\mathrm{His}_{6}{ }^{-}$ $m x i G$ were comparable to that of $m x i G^{-} / \mathrm{N}-\mathrm{His}_{6}-m x i G$, but degradation of the tagged protein was more pronounced (data not shown). Taken together, these data demonstrate that in the presence of $0.02 \%$ arabinose $\mathrm{His}_{6}-\mathrm{MxiG}$ is expressed at approximately wild-type levels and is able to participate in T3SS assembly and function much like wildtype MxiG. These growth conditions were thus used in all subsequent NC isolation trials.

\section{Affinity purification of NCs via His-tagged MxiG}

Purification of His-tagged NCs is described in detail in Methods. The novel part of the purification protocol was the introduction of the nickel affinity step. The His-tag mediated specific binding of $\mathrm{His}_{6}$-MxiG to Ni-NTA beads (Fig. 2). Examination of the elution fraction by SDS-PAGE and silver staining showed that four of the major bands detected were of the expected molecular masses for MxiD, MxiG, MxiJ and MxiH (Fig. 2, upper right panel). The strong enrichment in MxiG was also detected by immunoblotting (Fig. 2, lower panels). When $m x i G^{-} / \mathrm{C}^{-H_{i}}{ }_{6}-m x i G$ was used in similar experiments (not shown), a much poorer yield was obtained, suggesting that the MxiG Cterminus was not as accessible to the Ni-NTA beads or had poorer affinity for them. Hence, $m x i G^{-} / \mathrm{N}-\mathrm{His}_{6}-m x i G$ was used in all further trials.

A variety of non-ionic detergents were tested during the initial solubilization of spheroplasted bacteria (Blocker et al., 2001). Detergent choice and concentration had critical effects on enrichment and yield of NC preparations. The best non-ionic detergent conditions found were: $2.5 \%$ TX-100, $1 \%$ OG, $1 \%$ LAPAO, $1 \%$ DDAO and $1 \%$ NP-40 (not shown). Under all these conditions, four major bands were observed after SDS-PAGE and Coomassie staining, starting from only $200 \mathrm{ml}$ of bacterial culture, which Nterminal sequencing and/or immunoblotting identified as MxiD, $\mathrm{His}_{6}-\mathrm{MxiG}, \mathrm{MxiJ}$ and $\mathrm{MxiH}$. However, after extensive analysis of the minor bands seen in these two samples by mass spectrometry (MS), none of the expected other Ipa, Mxi or Spa proteins was identified (not shown). One other protein was identified that is not an expected NC component but has been previously indirectly implicated in T3SS-mediated virulence in Shigella flexneri, tRNA $N^{6}$ isopentenyladenosine $\left(\mathrm{i}^{6} \mathrm{~A} 37\right)$ synthetase (Durand et al., 1997).

The highest yield and the lowest number of contaminating bands were found for the TX-100 and OG preparations. 

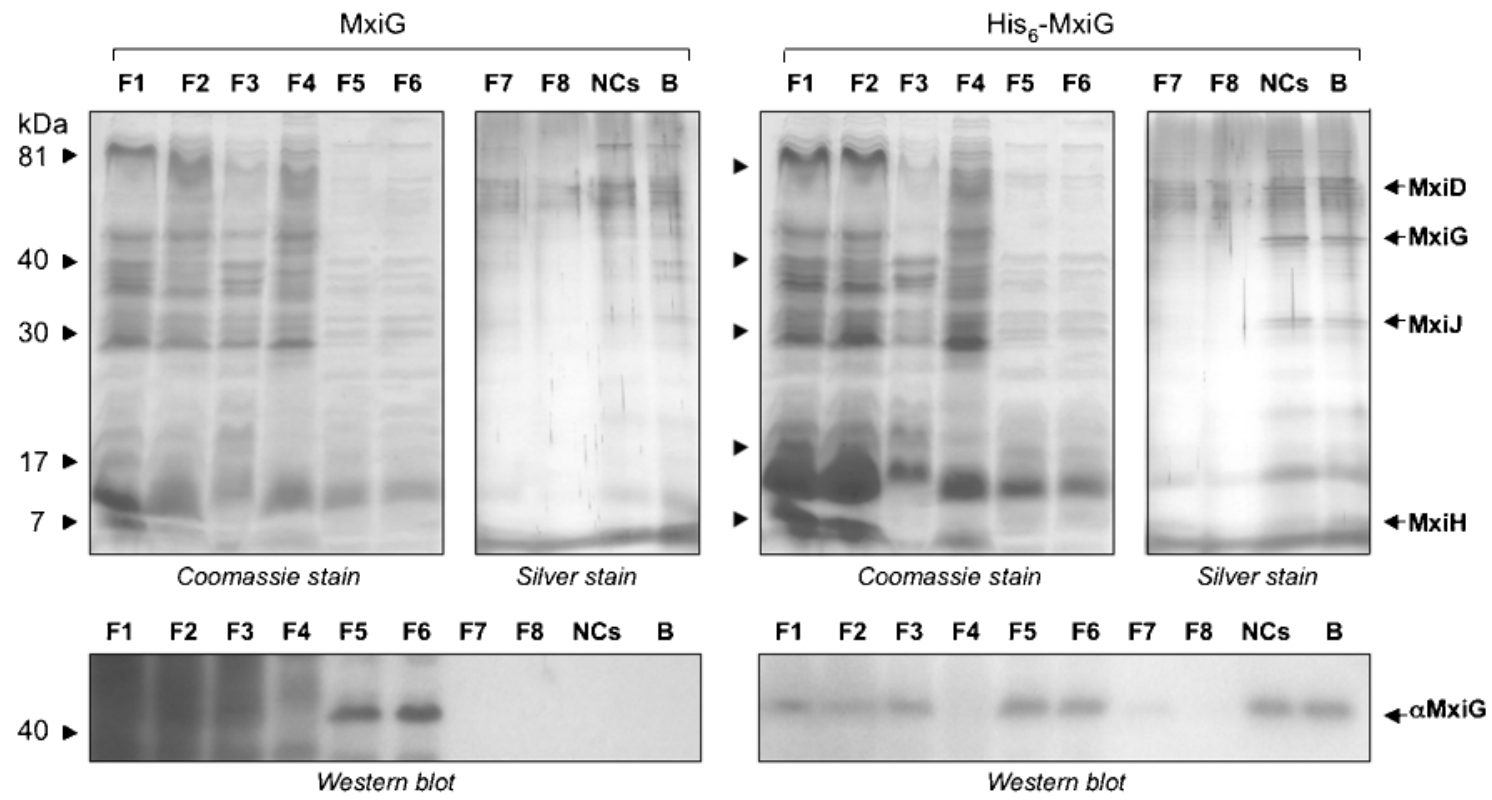

Fig. 2. Analysis of the steps in needle complex purification. $\mathrm{Ni}^{2+}$-affinity purification of NCs from $200 \mathrm{ml} m x i G^{-} / m x i G$ (MxiG) and $m x i G^{-} / \mathrm{N}-\mathrm{His}_{6}-m x i G\left(\mathrm{His}_{6}-\mathrm{MxiG}\right)$. Upper panels, the fractions (F1-F8, NCs and $\mathrm{B}$ ) corresponding to each step in the procedure using $1 \% \mathrm{TX}-100$, as outlined in Methods, were separated on SDS-polyacrylamide gels (15\%), which were cut in half and imaged after either Coomassie stain (for the early steps) or silver stain (for later fractions). Lower panels, the MxiG content of each of the fractions directly above was assessed after performing Western blots of SDS-polyacrylamide gels (10\%) using a mouse monoclonal anti-MxiG. On the extreme right-hand side, bands are marked which are found only in the preparation made from $m x i G^{-} / \mathrm{N}-\mathrm{His}_{6}-m x i G$ and correspond to MxiD, MxiG, MxiJ and MxiH.

Analysis of the EM images produced from these purifications reflected the purity of the samples seen by gel analysis (not shown). However, by EM the NCs in the OG sample seemed the least abundant, suggesting that OG still bound to the NCs prevented them from binding to grids efficiently. The LAPAO and DDAO purifications produced a large number of NCs, but these were found to be less stable upon further purification (not shown and see below). Hence, $2.5 \%$ TX-100 was used as a detergent in all subsequent purifications.

\section{Further NC purification by gel-filtration chromatography}

To ensure that no minor NC components were escaping detection because the preparations were insufficiently enriched, we scaled up our protocol. This yielded better NC preparations as judged by electron microscopy (Fig. 3a). We then added a final gel-filtration chromatography step. This last step took advantage of the unusually large size of NCs ( $\sim 4 \mathrm{MDa})$, which positioned them in the single fraction corresponding to the void volume of a Superdex 200 column (Fig. 3b). As shown in Fig. 3(c), the gel filtration step yielded NCs visualized as purified to near homogeneity, since only MxiD, MxiG, MxiJ and MxiH were visible on the gel by Coomassie stain. Silver staining of the entire purification allowed visualization of how this step separated NCs from most of the soluble proteins then found in the final fractions (compare the initial 'load', NC peak in ' $F_{8}$ ' and ' $F_{11-25}$ '). As shown in Fig. $3(\mathrm{~d})$, this further purification step did not damage NC morphology. In fact, once purified in this manner, the NCs could be preserved for months as outlined in Methods. Bands in $\mathrm{F}_{8}$ (Fig. 3c) that did not correspond by molecular mass to any previously identified NC components, but were seemingly enriched in the peak NC fraction, were selected for further analysis by MS (see Table 1). Besides the known Shigella NC components identified previously (Blocker et al., 2001) and above, the protein products of three ipa or mxilspa genes were identified in this preparation: IpaD, a known component of the needle distal tip complex (Espina et al., 2006; Sani et al., 2007b; Veenendaal et al., 2007), and two predicted minor NC inner-membrane proteins, Spa40 and Spa24, putative components of the central export apparatus of this T3SS.

\section{Validation of the novel compositional findings}

Although our aim was to identify especially the predicted minor inner membrane components of $\mathrm{NCs}$ in our improved preparations, directly validating the presence of such essential (Sukhan et al., 2001) yet very hydrophobic, minor and internal components of the $\mathrm{NC}$ is challenging. Sani et al. (2007a) reported identification of an additional 
(a)

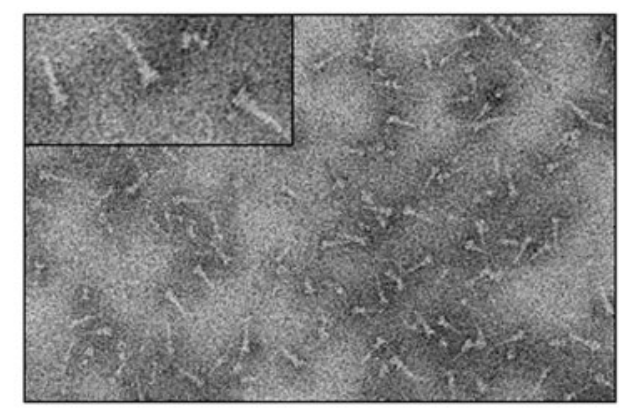

(b)

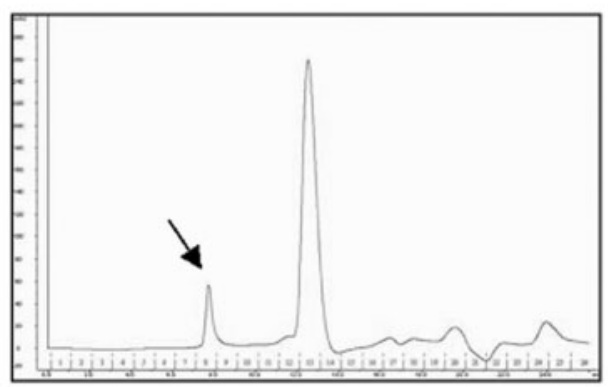

(c)

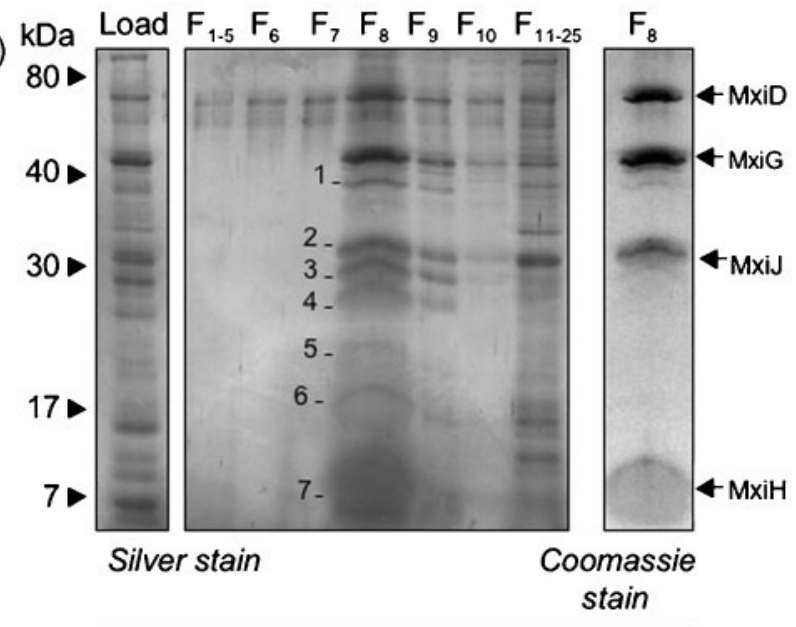

(d)

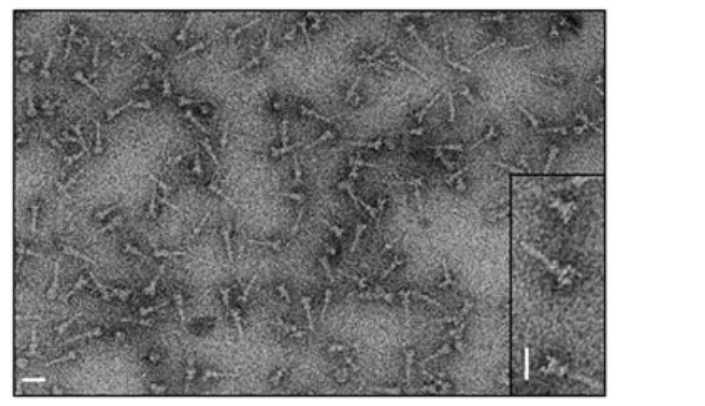

protein within their NC preparations, MxiM, the pilotinlike protein for the outer membrane component MxiD (Bayan et al., 2006). Even though we did not detect this protein upon SDS-PAGE separation followed by massspectrometry analysis of our final NC fraction, immunoblotting using an affinity-purified rabbit polyclonal antibody showed that it was clearly enriched there (Supplementary Fig. S1, available with the online version of this paper). Therefore we proceeded to use the antibody to test whether MxiM localized where we expected, i.e. on
Fig. 3. NC release with $2.5 \%(\mathrm{v} / \mathrm{v}) \mathrm{TX}-100$, followed by nickelaffinity isolation and size-exclusion chromatography. (a) Electron micrograph of the imidazole eluate of an NC preparation generated from 2 I of bacterial culture. Note that the eluate was diluted eightfold prior to grid preparation. Bars, $50 \mathrm{~nm}$. (b) Gel-filtration profile of the NC imidazole eluate on a Superdex 200 HR10/30 column. The arrow indicates the NC peak located in the void volume of the column. (c) SDS-PAGE analysis of the NC gel filtration run. About $2 \%$ of the sample load (initial imidazole eluate) and $25 \%$ of each of the indicated fractions were separated on a $15 \%$ gel, which was stained first with Coomassie (right), where only four major bands are seen corresponding from top to bottom to the molecular mass of MxiD, $\mathrm{His}_{6}-\mathrm{MxiG}, \mathrm{MxiJ}$ and $\mathrm{MxiH}$, and then with silver (left) using a kit compatible with MS (Invitrogen). Note that when the fractions are indicated as pooled, they were considered as only one fraction in terms of relative amounts loaded. The numbers close to the bands in the lane labelled ' $F_{8}$ ' indicate the bands that were subsequently analysed by MS (see Table 1). (d) Electron micrograph of undiluted NCs in fraction 8 (prior to concentration). Bars, $50 \mathrm{~nm}$.

the upper outer rim of the MxiD outer membrane ring of the NC. Using immunogold labelling, this protein was clearly detected as an intrinsic component of the NC outer membrane ring (Fig. 4). As both Spa24 and Spa40 were more abundant in our preparations than MxiM and also highly enriched there, we conclude that they too are bona fide NC components.

\section{DISCUSSION}

Using our new affinity isolation protocol and only $200 \mathrm{ml}$ of $m x i G^{-} / \mathrm{N}-\mathrm{His}_{6}-m x i G$ bacterial culture, highly enriched NCs can be easily obtained. This is 10-25-fold less starting material than all other published $\mathrm{NC}$ isolation procedures (Blocker et al., 2001; Daniell et al., 2001; Kimbrough \& Miller, 2000; Kubori et al., 1998; Marlovits et al., 2004; Ogino et al., 2006; Tamano et al., 2002). Scaling up this procedure to 21 cultures and adding an additional gel filtration step yields NCs purified to near homogeneity $\left(\sim 250 \mu \mathrm{l}\right.$ at $0.25 \mathrm{mg} \mathrm{ml}^{-1}$ or an estimated $15 \%$ of the NCs in the initial culture). This was never obtained before in even the best preparations of Marlovits et al. (2004) or Blocker et al. (2001), which were both contaminated with outer-membrane proteins. This far greater degree of enrichment has finally allowed detection of some of the expected minor NC components. In addition, our protocol uses near-physiological conditions throughout (no $\mathrm{pH}$ changes or $\mathrm{CsCl}$ gradients, unlike almost every other published procedure) and fewer high-speed centrifugation steps, which can only improve NC preservation. These samples were even suitable for long-term storage, which should increase the convenience of future high-resolution EM analysis.

We were surprised to find that the overnight 'leakage' and $\mathrm{CR}$ induction secretion profiles of the complemented $m x i G^{-}$strains did not exactly match those of the wild-type 
Table 1. MS data from numbered bands in $F_{8}$ of Fig. 3(c)

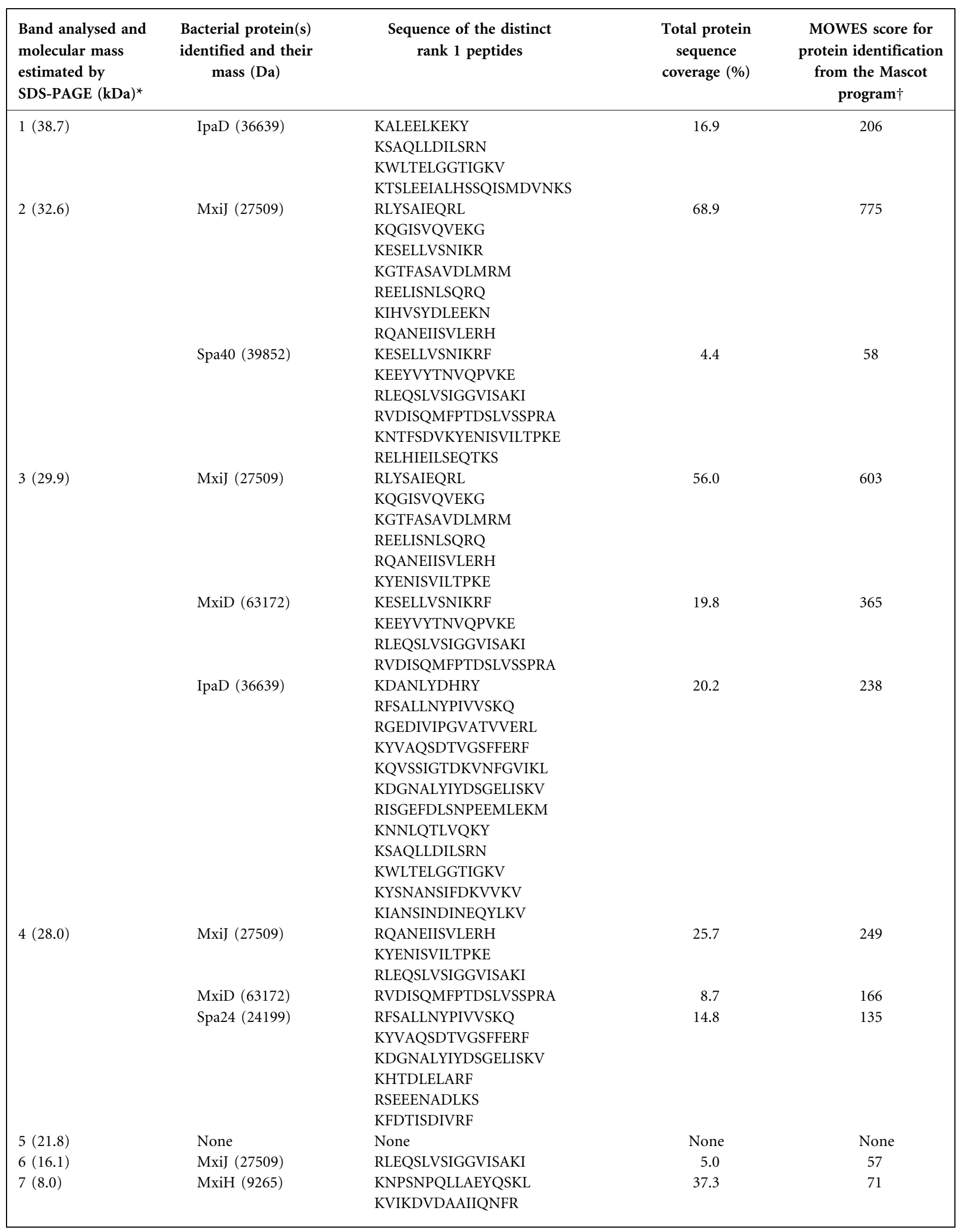


Table 1. cont.

${ }^{*}$ Measured from central part of band when it was large or twisted.

$\dagger$ After excision from the gel, silver stain removal and processing according to the manufacturer's instructions (Invitrogen), trypic protein digests were analysed by LC MS/MS using a Waters Q-TOF micro mass spectrometer. Searches on the resulting data were performed using Mascot, available at the Matrix Science web site (http://www.matrixscience.com). MOWES scores of above approximately 50 are within the $95 \%$ confidence interval for positive identification.

strain. At $0.02 \%$ arabinose, the overnight Ipa secretion pattern of $m x i G^{-} / m x i G$ corresponded approximately to that seen in the wild-type strain, but the overall protein level was substantially enhanced, and that of $m x i G^{-} / \mathrm{N}$ His $_{6}-m x i G$ displayed reduced IpaB and IpaA (Fig. 1b). In addition, at the same arabinose concentration, $m x i G^{-}$/ $m x i G$ was not inducible by $\mathrm{CR}$, while $m x i G^{-} / \mathrm{N}-\mathrm{His}_{6}-m x i G$ behaved much as wild-type (Fig. 1c). The reasons for these differences are not clear, especially as the expression levels of the MxiG protein from both constructs seemed similar (Fig. 2) and led to functional NC assembly, as evidenced by overnight 'leakage' of Ipa proteins. These phenomena may be linked to the short $\mathrm{N}$-terminal extension inserted in the untagged MxiG during the cloning process (see Methods). Nevertheless, they do suggest that a wild-type MxiG Nterminus is important in regulating proper low-level and inducible T3S.

Other than the previously known NC components, the only new potentially relevant protein identified in merely affinity-purified NC preparations, $i^{6} \mathrm{~A} 37$ synthetase, is implicated in post-transcriptionally enhancing expression of T3SS-encoding genes in S. flexneri (Durand et al., 1997). No new Ipa, Mxi or Spa proteins were identified in such NC preparations. However, some such components, and not $i^{6} \mathrm{~A} 37$ synthetase, were identified after further purification of these preparations using gel filtration. Therefore, we

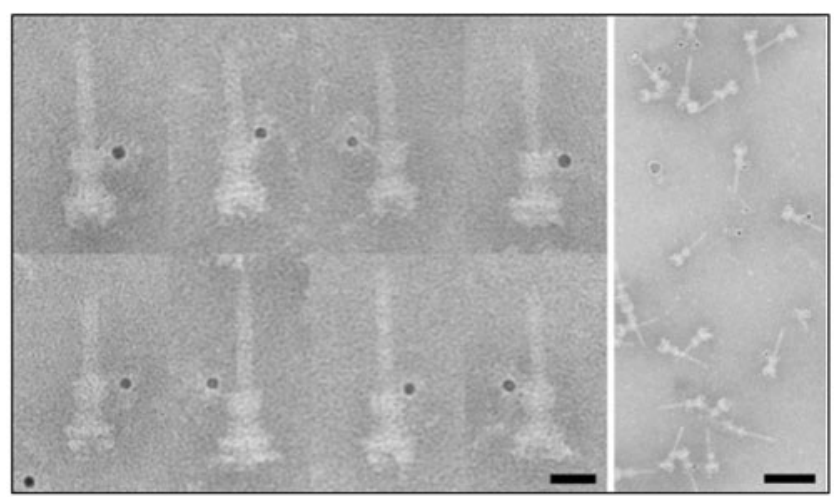

Fig. 4. Immunogold localization of MxiM within purified NC. Left, gallery of electron micrographs of NCs decorated with anti-MxiM affinity purified primary $A b$ followed by $5 \mathrm{~nm}$ gold-conjugated secondary $\mathrm{Ab}$ and then negatively stained. Right, field overview of the same electron micrographs. The outer membrane ring of NCs is specifically decorated. Scale bars are $100 \mathrm{~nm}$ and $200 \mathrm{~nm}$, respectively. conclude that (i) $i^{6} \mathrm{~A} 37$ synthetase is not a true $\mathrm{NC}$ component and (ii) the predicted NC components had not all been extracted by the detergents used, but instead were present in too low a copy number for detection without prior NC enrichment to this level.

The presence within NCs of IpaD, recently identified as part of the needle tip complex, is no longer a surprise (Espina et al., 2006; Sani et al., 2007b; Veenendaal et al., 2007). However, $\mathrm{IpaD}(36.6 \mathrm{kDa})$ was the only component identified in band 1 (migrating at an estimated $38.7 \mathrm{kDa}$, see Supplementary data) which was easily visualized by silver staining and more poorly detectable by Coomassie staining. This gives an estimate of the detection limits of our current procedure, since we expect four copies of IpaD per NC (Deane et al., 2006; Johnson et al., 2007; Veenendaal et al., 2007). Previously, using Amido black staining, which is approximately as sensitive as Coomassie staining, we were only able to reliably detect large components present in more than approximately 20 copies per NC (Blocker et al., 2001). At the time, we only detected Mxil, the minor periplasmic rod component, because it comigrated with the major component MxiH by SDS-PAGE. MxiI was not found in even the most enriched NC preparations during the present work, possibly because MS was used here instead of $\mathrm{N}$-terminal sequencing and small proteins generate few tryptic peptides.

Pilotin-like proteins, such as MxiM, normally interact with their outer membrane secretins in a 1:1 molar ratio (Bayan et al., 2006). Therefore, MxiM should have been easily detectable in our preparations as MxiD is thought to be present there in approximately 10-20 copies (Blocker et al., 2001; Marlovits et al., 2004). However, we only detected it in our most enriched fractions and only by immunoblotting. We suspect that its presence in substoichiometric amounts is due to the high concentration of TX-100 used in our isolation procedure. Indeed, pilotin-secretin interactions might be labile, especially in detergents. Nevertheless, it is the first time that a protein of this type has been localized within a native and finalized secretionrelated structure.

Spa40 and Spa24, predicted components of the T3SS inner membrane export apparatus, were both detected in our new NC preparations. Knockout of their gene homologues within the region encoding the closely related Salmonella SPI1 T3SS leads to secretons without needles (Sukhan et al., 2001) and decreased detection of the MxiJ homologue. However, this is believed to be the first time that these highly conserved components have been detected in any 
NC preparation, although the flagellar homologue of Spa24, FliP, has been previously detected in basal bodies purified to near homogeneity (Fan et al., 1997; Jones \& Macnab, 1990; Jones et al., 1990). Spa40 (39.9 kDa) was found in a band that contained mostly MxiJ $(27.5 \mathrm{kDa})$, band 2, which migrated at $32.6 \mathrm{kDa}$. MxiJ is lipidated, likely at its $\mathrm{N}$ terminus (Allaoui et al., 1992; Yip et al., 2005), perhaps explaining its migration at a molecular mass higher than expected. As Spa40 migrated at a molecular mass substantially lower than predicted, it might be degraded or processed in our NC preparations in an analogous manner to its homologues in the flagellum (Ferris et al., 2005) and Yersinia T3SS (Lavander et al., 2002). Only one peptide belonging to Spa40 was identified, which, given the substantial size of the protein, suggests that it is present in extremely low amounts in NCs. Indeed, in flagella, FlhB is proposed to be present in a one-to-one ratio with FliR (Van Arnam et al., 2004), which is itself probably present in a single copy per basal body (Fan et al., 1997).

Three peptides were identified for Spa24 $(24.2 \mathrm{kDa})$ in a band migrating at $28 \mathrm{kDa}$. As the signal peptide of the flagellar homologue of Spa24, FliP, is known to be cleaved (Ohnishi et al., 1997), we cannot presently explain why we find it at a higher than expected molecular mass in our SDS-polyacrylamide gels. This could be due to its very hydrophobic nature, but the possibility that it is lipidated and/or glycosylated should be also considered. The greater number of peptides recovered for this smaller protein suggests that it may be present in higher copy number than Spa40. Indeed, FliP is estimated to be present in four or five copies per flagellar basal body (Fan et al., 1997). Although stoichiometries cannot be directly inferred from mass spectrometry data, these initial notions of protein abundance are supported by the fact that we only detected Spa40 and Spa24 in our most enriched fractions and by analysis of symmetry and mass of NC subdomains using high-resolution EM of negatively stained samples and scanning transmission EM. These latter two lines of evidence suggest that MxiG and MxiJ do indeed constitute over $90 \%$ of the average mass of the NC inner membrane region (J. L. Hodgkinson, D. Stabat, M. Simons, J. Wall \& A. J. Blocker, unpublished).

Both Spa40/FlhB and Spa24/FliP are expected to localize to the central 'plate' or 'rod' in the inner membrane of their respective T3SSs (Blocker et al., 2001; Francis et al., 1994; Sosinsky et al., 1992b). For NCs, this structure has recently been termed the 'socket' and shown not to be composed of either the needle or the periplasmic needle rod components (Marlovits et al., 2006; Sani et al., 2007a). FliR has been shown to localize there immunologically and it can be fused to FlhB and still lead to the assembly of functional flagella, suggesting that FlhB is also found in the central export apparatus (Ohnishi et al., 1997). As these central structures are so small, however, visualizing them properly, as well as assessing their integrity and component stoichiometries, especially following necessary detergent extraction, remain major challenges for the future.
Further biochemical work is also required to test for the presence of the remaining expected, but so far unidentified, NC components (MxiA, Spa29 and Spa9). Finally, quite a few of the remaining peptides identified from a number of the lower molecular mass bands belonged to the major NC components MxiD, MxiG and MxiJ (Table 1). This is probably caused by partial degradation of the NCs during purification (Fig. 3a, 3d). It may be that NCs are more fragile than flagellar basal bodies, which seem to resist harsher purification conditions (Jones et al., 1990). Due to $\mathrm{NC}$ instability, the stoichiometries of the entire set of NC components can not be accurately resolved by biochemical studies, which are made on particle populations (Jones et al., 1990; Marlovits et al., 2004). Rather, mass analysis by scanning transmission EM (Sosinsky et al., 1992a), where only intact structures or substructures can be selected for measurement, should be used.

\section{ACKNOWLEDGEMENTS}

S.F.Z. was supported by a Studienstiftung des deutschen Volkes scholarship, and D. S. and J. L. H. by MRC Project Grant G0401595 to A. J.B. A. K. J.V. is funded by an EC Marie Curie Postdoctoral fellowship (MEIF-CT-2005-023694). S. J. was supported by the Guy G. F. Newton Senior Research Fellowship to A. J. B. and MRC Project Grant G0400389 to Dr Susan M. Lea. S.F.Z. acknowledges Michael Hensel (FAU Erlangen-Nürnberg) for supervision and grading of his diploma project. Frank Ebel is acknowledged for assistance in making the anti-MxiG antibody while at the Institut Pasteur, Paris. Tony Willis (MRC Immunochemistry Unit, Biochemistry, Oxford) and Benjamin Thomas (Central Proteomics Facility, SWDSOP) are thanked for N-terminal sequencing and MS analysis, respectively. EM analysis was performed within the SWDSOP Bio-Imaging facilities, with the help of Mike Shaw. Tohru Minamino is thanked for recommending the appropriate flagellar reading and for discussion of its implications for NCs. Keiichi Namba is thanked for suggesting trehalose as a cryopreservant. Tony Maurelli is thanked for providing the E. coli strain expressing recombinant MxiM and the MxiM antiserum.

\section{REFERENCES}

Allaoui, A., Sansonetti, P. J. \& Parsot, C. (1992). MxiJ, a lipoprotein involved in secretion of Shigella Ipa invasins, is homologous to YscJ, a secretion factor of the Yersinia Yop proteins. J Bacteriol 174, 7661-7669.

Allaoui, A., Sansonetti, P. J. \& Parsot, C. (1993). MxiD, an outer membrane protein necessary for the secretion of the Shigella flexneri Ipa invasins. Mol Microbiol 7, 59-68.

Allaoui, A., Sansonetti, P. J., Menard, R., Barzu, S., Mounier, J., Phalipon, A. \& Parsot, C. (1995). MxiG, a membrane protein required for secretion of Shigella spp. Ipa invasins: involvement in entry into epithelial cells and in intercellular dissemination. Mol Microbiol 17, 461-470.

Bahrani, F. K., Sansonetti, P. J. \& Parsot, C. (1997). Secretion of Ipa proteins by Shigella flexneri: inducer molecules and kinetics of activation. Infect Immun 65, 4005-4010.

Bayan, N., Guilvout, I. \& Pugsley, A. P. (2006). Secretins take shape. Mol Microbiol 60, 1-4.

Benjelloun-Touimi, Z., Sansonetti, P. J. \& Parsot, C. (1995). SepA, the major extracellular protein of Shigella flexneri: autonomous 
secretion and involvement in tissue invasion. Mol Microbiol 17, 123-135.

Blocker, A., Gounon, P., Larquet, E., Niebuhr, K., Cabiaux, V., Parsot, C. \& Sansonetti, P. (1999). The tripartite type III secreton of Shigella flexneri inserts IpaB and IpaC into host membranes. J Cell Biol 147, 683-693.

Blocker, A., Jouihri, N., Larquet, E., Gounon, P., Ebel, F., Parsot, C., Sansonetti, P. \& Allaoui, A. (2001). Structure and composition of the Shigella flexneri 'needle complex', a part of its type III secreton. Mol Microbiol 39, 652-663.

Blocker, A., Komoriya, K. \& Aizawa, S. (2003). Type III secretion systems and bacterial flagella: insights into their function from structural similarities. Proc Natl Acad Sci U S A 100, 3027-3030.

Burghout, P., van Boxtel, R., Van Gelder, P., Ringler, P., Muller, S. A., Tommassen, J. \& Koster, M. (2004). Structure and electrophysiological properties of the $\mathrm{YscC}$ secretin from the type III secretion system of Yersinia enterocolitica. J Bacteriol 186, 4645-4654.

Cordes, F. S., Komoriya, K., Larquet, E., Yang, S., Egelman, E. H., Blocker, A. \& Lea, S. M. (2003). Helical structure of the needle of the type III secretion system of Shigella flexneri. J Biol Chem 278, 17103-17107.

Cordes, F. S., Daniell, S., Kenjale, R., Saurya, S., Picking, W. L., Picking, W. D., Booy, F., Lea, S. M. \& Blocker, A. (2005). Helical packing of needles from functionally altered Shigella type III secretion systems. J Mol Biol 354, 206-211.

Cossart, P. \& Sansonetti, P. J. (2004). Bacterial invasion: the paradigms of enteroinvasive pathogens. Science 304, 242-248.

Daniell, S. J., Takahashi, N., Wilson, R., Friedberg, D., Rosenshine, I., Booy, F. P., Shaw, R. K., Knutton, S., Frankel, G. \& Aizawa, S. (2001). The filamentous type III secretion translocon of enteropathogenic Escherichia coli. Cell Microbiol 3, 865-871.

Deane, J. E., Roversi, P., Cordes, F. S., Johnson, S., Kenjale, R., Daniell, S., Booy, F., Picking, W. D., Picking, W. L. \& other authors (2006). Molecular model of a type III secretion system needle: implications for host-cell sensing. Proc Natl Acad Sci U S A 103, 12529-12533.

Durand, J. M., Bjork, G. R., Kuwae, A., Yoshikawa, M. \& Sasakawa, C. (1997). The modified nucleoside 2-methylthio- $N^{6}$-isopentenyladenosine in tRNA of Shigella flexneri is required for expression of virulence genes. J Bacteriol 179, 5777-5782.

Espina, M., Olive, A. J., Kenjale, R., Moore, D. S., Ausar, S. F., Kaminski, R. W., Oaks, E. V., Middaugh, C. R., Picking, W. D. \& Picking, W. L. (2006). IpaD localizes to the tip of the type III secretion system needle of Shigella flexneri. Infect Immun 74, 4391-4400.

Fan, F., Ohnishi, K., Francis, N. R. \& Macnab, R. M. (1997). The FliP and FliR proteins of Salmonella typhimurium, putative components of the type III flagellar export apparatus, are located in the flagellar basal body. Mol Microbiol 26, 1035-1046.

Ferris, H. U., Furukawa, Y., Minamino, T., Kroetz, M. B., Kihara, M., Namba, K. \& Macnab, R. M. (2005). FlhB regulates ordered export of flagellar components via an autocleavage mechanism. J Biol Chem 280, 41236-41242.

Francis, N. R., Sosinsky, G. E., Thomas, D. \& DeRosier, D. J. (1994). Isolation, characterization and structure of bacterial flagellar motors containing the switch complex. J Mol Biol 235, 1261-1270.

Hale, T. L. (1998). Bacilliary dysentery. In Topley and Wilson's Microbiology and Microbial Infections, pp. 479-493. Edited by A. Balows, M. Sussman \& L. H. Collier. London: Hodder Arnold.

Hirai, T., Murata, K., Mitsuoka, K., Kimura, Y. \& Fujiyoshi, Y. (1999). Trehalose embedding technique for high-resolution electron crystallography: application to structural study on bacteriorhodopsin. J Electron Microsc (Tokyo) 48, 653-658.
Johnson, S., Roversi, P., Espina, M., Olive, A., Deane, J. E., Birket, S., Field, T., Picking, W. D., Blocker, A. J. \& other authors (2007). Self-chaperoning of the type III secretion system needle tip proteins IpaD and BipD. J Biol Chem 282, 4035-4044.

Jones, C. J. \& Macnab, R. M. (1990). Flagellar assembly in Salmonella typhimurium: analysis with temperature-sensitive mutants. J Bacteriol 172, 1327-1339.

Jones, C. J., Macnab, R. M., Okino, H. \& Aizawa, S. (1990). Stoichiometric analysis of the flagellar hook-(basal-body) complex of Salmonella typhimurium. J Mol Biol 212, 377-387.

Kenjale, R., Wilson, J., Zenk, S. F., Saurya, S., Picking, W. L., Picking, W. D. \& Blocker, A. (2005). The needle component of the type III secreton of Shigella regulates the activity of the secretion apparatus. J Biol Chem 280, 42929-42937.

Kimbrough, T. G. \& Miller, S. I. (2000). Contribution of Salmonella typhimurium type III secretion components to needle complex formation. Proc Natl Acad Sci U S A 97, 11008-11013.

Kubori, T., Matsushima, Y., Nakamura, D., Uralil, J., Lara-Tejero, M., Sukhan, A., Galan, J. E. \& Aizawa, S. I. (1998). Supramolecular structure of the Salmonella typhimurium type III protein secretion system. Science 280, 602-605.

Lavander, M., Sundberg, L., Edqvist, P. J., Lloyd, S. A., Wolf-Watz, H. \& Forsberg, A. (2002). Proteolytic cleavage of the FlhB homologue YscU of Yersinia pseudotuberculosis is essential for bacterial survival but not for type III secretion. J Bacteriol 184, 4500-4509.

Magdalena, J., Hachani, A., Chamekh, M., Jouihri, N., Gounon, P., Blocker, A. \& Allaoui, A. (2002). Spa32 regulates a switch in substrate specificity of the type III secreton of Shigella flexneri from needle components to Ipa proteins. J Bacteriol 184, 3433-3441.

Marlovits, T. C., Kubori, T., Sukhan, A., Thomas, D. R., Galan, J. E. \& Unger, V. M. (2004). Structural insights into the assembly of the type III secretion needle complex. Science 306, 1040-1042.

Marlovits, T. C., Kubori, T., Lara-Tejero, M., Thomas, D., Unger, V. M. \& Galan, J. E. (2006). Assembly of the inner rod determines needle length in the type III secretion injectisome. Nature 441, 637-640.

Morita-Ishihara, T., Ogawa, M., Sagara, H., Yoshida, M., Katayama, E. \& Sasakawa, C. (2006). Shigella Spa33 is an essential C-ring component of type III secretion machinery. J Biol Chem 281, 599-607.

Niebuhr, K. \& Ebel, F. (2003). Generation of monoclonal antibodies against secreted proteins of STEC. Methods Mol Med 73, 125-135.

Ogino, T., Ohno, R., Sekiya, K., Kuwae, A., Matsuzawa, T., Nonaka, T., Fukuda, H., Imajoh-Ohmi, S. \& Abe, A. (2006). Assembly of the type III secretion apparatus of enteropathogenic Escherichia coli. J Bacteriol 188, 2801-2811.

Ohnishi, K., Fan, F., Schoenhals, G. J., Kihara, M. \& Macnab, R. M. (1997). The FliO, FliP, FliQ, and FliR proteins of Salmonella typhimurium: putative components for flagellar assembly. J Bacteriol 179, 6092-6099.

Parsot, C. (1994). Shigella flexneri: genetics of entry and intercellular dissemination in epithelial cells. Curr Top Microbiol Immunol 192, 217-241.

Sani, M., Allaoui, A., Fusetti, F., Oostergetel, G. T., Keegstra, W. \& Boekema, E. J. (2007a). Structural organization of the needle complex of the type III secretion apparatus of Shigella flexneri. Micron 38, 291-301.

Sani, M., Botteaux, A., Parsot, C., Sansonetti, P., Boekema, E. J. \& Allaoui, A. (2007b). IpaD is localized at the tip of the Shigella flexneri type III secretion apparatus. Biochim Biophys Acta 1770, 307-311.

Schuch, R. \& Maurelli, A. T. (1999). The Mxi-Spa type III secretory pathway of Shigella flexneri requires an outer membrane lipoprotein, MxiM, for invasin translocation. Infect Immun 67, 1982-1991. 
Schuch, R. \& Maurelli, A. T. (2001). MxiM and MxiJ, base elements of the Mxi-Spa type III secretion system of Shigella, interact with and stabilize the MxiD secretin in the cell envelope. J Bacteriol 183, 6991-6998.

Sosinsky, G. E., Francis, N. R., DeRosier, D. J., Wall, J. S., Simon, M. N. \& Hainfeld, J. (1992a). Mass determination and estimation of subunit stoichiometry of the bacterial hook-basal body flagellar complex of Salmonella typhimurium by scanning transmission electron microscopy. Proc Natl Acad Sci U S A 89, 4801-4805.

Sosinsky, G. E., Francis, N. R., Stallmeyer, M. J. \& DeRosier, D. J. (1992b). Substructure of the flagellar basal body of Salmonella typhimurium. J Mol Biol 223, 171-184.

Sukhan, A., Kubori, T., Wilson, J. \& Galan, J. E. (2001). Genetic analysis of assembly of the Salmonella enterica serovar Typhimurium type III secretion-associated needle complex. J Bacteriol 183, 1159-1167.

Suzuki, T., Lett, M. C. \& Sasakawa, C. (1995). Extracellular transport of VirG protein in Shigella. J Biol Chem 270, 30874-30880.
Tamano, K., Aizawa, S. \& Sasakawa, C. (2002). Purification and detection of Shigella type III secretion needle complex. Methods Enzymol 358, 385-392.

Van Arnam, J. S., McMurry, J. L., Kihara, M. \& Macnab, R. M. (2004). Analysis of an engineered Salmonella flagellar fusion protein, FliRFlhB. J Bacteriol 186, 2495-2498.

Veenendaal, A. K., Hodgkinson, J. L., Schwarzer, L., Stabat, D., Zenk, S. F. \& Blocker, A. J. (2007). The type III secretion system needle tip complex mediates host cell sensing and translocon insertion. Mol Microbiol 63, 1719-1730.

Yip, C. K., Kimbrough, T. G., Felise, H. B., Vuckovic, M., Thomas, N. A., Pfuetzner, R. A., Frey, E. A., Finlay, B. B., Miller, S. I. \& other authors (2005). Structural characterization of the molecular platform for type III secretion system assembly. Nature 435, 702-707.

Edited by: T. Palmer 